


\title{
INTERFERENCIAS FONÉTICAS-FONOLÓGICAS EN RELATOS DE VIDA DE INMIGRANTES PORTUGUESES
}

\author{
PHONETIC-PHONOLOGICAL INTERFERENCES IN LIFE \\ STORIES OF PORTUGUESE IMMIGRANTS
}

\author{
Viana Prato Duarte \\ Inmaculada Tamarit Vallés
}

\begin{abstract}
RESUMEN
Este trabajo de investigación trata sobre las interferencias fonéticas-fonológicas presentes en relatos de vida de inmigrantes portugueses en Venezuela. La utilidad de este estudio radica en el hecho de que el fenómeno migratorio, en el que dos culturas se encuentran, influyéndose mutuamente, es universal; por lo que la comprensión del principal vehículo integrador como lo es la lengua debe ser objeto de estudio minucioso. Nuestro objetivo de investigación es clasificar las interferencias lingüísticas en el discurso oral de los inmigrantes portugueses que llegaron a Venezuela en el periodo 1950 a 1980, conforme a la clasificación de las interferencias fonéticas-fonológicas de Porto (2009), Niño (2007) y Obediente (2007). Utilizamos un cuestionario y la técnica de la entrevista. La muestra estuvo constituida por 26 sujetos elegidos al azar. Utilizamos el Software Atlas ti versión 8.0 para identificar la frecuencia de las interferencias fonéticas-fonológicas en los relatos de vida. Las interferencias fonéticas-fonológicas más recurrentes fueron las de sustitución de vocablos con un $55 \%$ de frecuencia, las de reducción de fonemas con $25 \%$ y finalmente con un $20 \%$ las de incrementación de sonidos. Concluimos que ocurrió un estancamiento en la evolución de la interlengua, es decir, se evidencia una fosilización de L2.

Palabras clave: interferencias fonéticas-fonológicas; interlengua; fosilización; inmigrantes portugueses; relatos de vida.
\end{abstract}

\begin{abstract}
This research is about the phonetic-phonological interferences present in the life stories of Portuguese immigrants in Venezuela. The usefulness of this study lies in the fact that the migratory phenomenon, in which two cultures meet and influence one another, is universal, so the understanding of the main integrative vehicle as the language must be carefully studied. Our research objective is to classify linguistic interference in the oral discourse of Portuguese immigrants who arrived in Venezuela in the period 1950 to 1980 , according to the classification of phonetic-phonological interferences of Porto (2009), Niño (2007) and Obediente (2007). We use a questionnaire and interview techniques. The sample consisted of 26 randomly chosen subjects. We use the Atlas Ti software version 8.0 to identify the frequency of phonetic-phonological interferences in life stories. The most recurrent phonetic-phonological interferences were those involving the substitution of words with a frequency of $55 \%$, those involving the reduction of phonemes by $25 \%$ and finally, those with a $20 \%$ increase in sounds. We conclude that there was a stalemate in the evolution of the interlanguage, i.e. evidence of a fossilization of L2.

Keywords: phonetic-phonological interference; interlanguage; fossilization; Portuguese immigrants; life stories.
\end{abstract}

\footnotetext{
Mag. Viana Prato Duarte. Profesora Asociada del Departamento de Idiomas. Universidad Nacional Experimental Francisco de Miranda. Falcón, Venezuela.

Correo electrónico: vianaprato@gmail.com

Dra. Inmaculada Tamarit Vallés. Profesora Titular del Departamento de Lingüística Aplicada. Universitat Politècnica de Valéncia. Valencia, España.

Correo electrónico: itamarit@upv.es

Recepción: 12- 06- 19

Aceptación: 29-10- 19
} 


\section{Introducción}

La interferencia fonética es uno de los problemas más comunes en el proceso de aprendizaje de un idioma extranjero. La introducción de elementos fonológicos foráneos de un sistema lingüístico a otro puede ser motivo de interferencia. Asimismo, hay que tener en cuenta que el contacto de dos lenguas diferentes puede favorecer la aparición de fonemas con rasgos articulatorios de tipo muy cercano. En el caso del español y el portugués, conviene subrayar el grado de afinidad entre ambas lenguas que entran en contacto por las proximidades territoriales de Portugal con España y de Brasil con países vecinos. Este trabajo de investigación trata precisamente sobre las interferencias fonéticas observadas entre los inmigrantes portugueses que viajaron a Venezuela entre 1950 y 1980, lo cual puso en contacto dos culturas con idiomas muy cercanos lingüísticamente.

En palabras de Pessini (2003, p. 76, en Téis, 2007, p. 74), el portugués y el español son lenguas románicas muy cercanas, por tanto, el intercambio de conocimientos de una a otra puede alcanzar idealmente más del $90 \%$. Esta proximidad entre los dos idiomas es una desventaja sobre todo en el nivel fonético-fonológico, porque el aprendiz busca en su lengua materna estructuras semejantes con una pronunciación aproximada, por lo que se aleja más de la pronunciación correcta de la lengua (Malaca y Reis, 2007). Es decir, "a interferência fonológica da língua materna na língua alvo, na maioria dos casos, permanece para sempre, mesmo quando o aprendente já adquiriu total domínio sobre o vocabulário e a gramática da língua estrangeira"1 (Malaca y Reis, 2007, p. 2).

En cuanto al tiempo de contacto entre dos culturas, las interferencias generadas por un acercamiento prolongado entre dos o varias comunidades lingüísticas pueden afectar la producción de los sonidos en ambas culturas. En este orden de ideas, Cambrussi, Ferreira y Gritti (2009, p. 71) consideran además que estas interferencias fonológicas de la lengua materna en la lengua que se aprende permanecen a lo largo de toda la vida del hablante, independientemente de su nivel de dominio de la lengua extranjera. Justamente en este estudio, las interferencias que describimos son aquellas que aparecen después de más de 40 años de encuentro de dos culturas con lenguas diferentes.

En nuestra opinión, los estudios acerca del comportamiento de estas lenguas en situación de contacto son escasos. No obstante, a continuación presentamos algunos trabajos que relacionan estos dos idiomas.

Godoy, Moreira, Rodríguez y Roppel (1994) elaboraron un manual sobre la pronunciación del español específico para brasileños en el que presentaron cuadros fonéticos y fonológicos del castellano y del portugués brasileño, acompañados de explicaciones y consideraciones sobre la norma y las variantes dialectales (regionales y sociales). En su tesis también incorporan la proposición de ejercicios para trabajar las distinciones fonológicas y fonéticas, que incluyen cuadros contrastivos que servirán para indicar los fonemas y sonidos comunes, los semejantes y los diferentes entre las lenguas. Para la clasificación de las interferencias fonéticas-fonológicas en nuestro estudio, nos guiamos por su trabajo de investigación. 
Blanco (2002), en su estudio "Una propuesta fonética didáctica para brasileños estudiantes de español", señala una serie de problemas de pronunciación de los lusohablantes al momento del aprendizaje del español. El autor plantea que los problemas de pronunciación que se suelen dar con más frecuencia en la producción oral de los estudiantes brasileños de español son:

- $\quad$ el luso hablante tenderá hacia la abertura de las vocales medias e, o

- la pronunciación de la letra $\mathrm{v}$ en vez de b

- la palatalización de las consonantes t y d ante las vocales e, i

- la palatalización de la g y la j o bien las pronunciará como una r, debido a que no consiguen la articulación velar de estas dos consonantes

- la velarización de la r vibrante múltiple

- la pronunciación de la s como un zumbido cuando aparece entre vocales al realizarla como si fuera la s sonora portuguesa, a una fortísima nasalidad transportada de su lengua materna

- la inserción de la vocal i, delante de ciertos grupos consonánticos

Con base en los problemas de pronunciación constatados en el estudio de Blanco, consideramos importante tener en cuenta esas dificultades y compararlas con las observadas en los relatos de vida de los inmigrantes portugueses en Venezuela, ya que este estudio aporta a nuestro trabajo conocimientos valiosos acerca de las interferencias fonéticas posibles entre estas dos lenguas emparentadas.

Férriz (2001) realiza un análisis contrastivo de los sistemas fonológicos del portugués (europeo y brasileño) y del español. En su trabajo, señala una caracterización fónica de la interlengua de castellanohablantes adultos aprendientes de portugués basada en fenómenos de transferencia. Su trabajo describe los sistemas fonológicos del portugués europeo y brasileño, los cuales son útiles para poder clasificar las interferencias fonéticas presentes en los inmigrantes portugueses llegados a Venezuela alrededor de los años 1950 a 1980. En líneas generales, esta investigación contribuye de manera importante ante la escasez de publicaciones especializadas relacionadas con la descripción de los sistemas fonéticos del español y del portugués.

Tavares (2006) expone que son abundantes las interferencias fonéticas-fonológicas entre el español y el portugués. Este trabajo expone las razones por las que se produjo el fenómeno de contacto lingüístico entre las lenguas española y portuguesa en Venezuela por los inmigrantes portugueses arraigados en el país. Concluye que, a pesar de que la adquisición haya sido espontánea o dirigida, en la niñez o en la adultez, siempre saldrán a flote interferencias de la L1 sobre la L2 -y viceversa- que el individuo tenga.

Brisolara (2011, p. 169) plantea que el portugués y el español poseen semejanzas. Aunque presentan diferencias significativas en lo referente a los aspectos fonético-fonológicos, existen asimismo varias particularidades comunes no solo fonético-fonológicas sino también morfológicas, sintácticas y semánticas. Estos rasgos que caracterizan ambas lenguas hacen que el hablante nativo del portugués, al aprender español, produzca una serie de interferencias de su Lengua Materna (LM) en la Lengua Extranjera (LE). Las interferencias de naturaleza fonético-fonológicas son bastante frecuentes en la adquisición del español como LE, de modo especial y con características específicas por hablantes de lengua portuguesa. En suma, este estudio destaca que los alumnos de todos los niveles de escolaridad presentan algunas interferencias de su Lengua Materna (portugués) en la Lengua Extranjera (español). 
En contraste con los estudios anteriores, Abreu (2011) estudia las interferencias fonéticas-fonológicas del portugués hablado por inmigrantes libaneses. El autor aclara que las lenguas en contacto producen interferencias y concluye que las interferencias se producen por el distanciamiento entre los dos sistemas fonético-fonológicos (portugués y libanes) y por la ausencia de un aprendizaje formal del portugués. Sería entonces, según este autor, el distanciamiento fonológico de los idiomas lo que acentuaría las interferencias y no su proximidad.

De manera semejante, otro autor describe las interferencias fonéticas de dos lenguas distantes confirmando los señalamientos de Abreu (2011). Elsaadani (2016), en su investigación "Analyse des interférences phonologiques entre l'arabe et le français: étude contrastive", describe el sistema fonético de ambas lenguas con el fin de precisar las causas de los errores fonológicos. El autor evidencia la presencia de interferencias fonéticas entre el francés y el árabe porque estas lenguas no poseen exactamente los mismos sonidos, es decir que son dos sistemas fonéticos muy diferentes.

En lo que respecta a las interferencias fonéticas observadas en inmigrantes portugueses que llegaron a Venezuela en los años 1950 a 1980, debemos, en primer lugar, señalar algunos aspectos resaltantes de esta migración. La inmigración de portugueses en Venezuela ha presentado varios momentos importantes en la historia venezolana. Tres décadas (1950 a 1980) han sido especialmente significativas por su magnitud y su influencia en la cultura actual venezolana. La inmigración portuguesa se duplicó durante los años 50, alcanzando un $9 \%$ del total de la inmigración en Venezuela para 1961. A partir de los 80, el porcentaje de inmigrantes es todavía mayor al sumar los indocumentados (González-Ordosgoitti, 1991). La religión, los lugares de encuentro y las actividades económicas de los inmigrantes portugueses determinaron en gran medida el modo de su integración en Venezuela. Estos componentes interculturales poseen un alto impacto en lo referente a las variaciones lingüísticas. La variedad de español hablado en Venezuela es un factor decisivo para las consecuencias lingüísticas de ese contacto, consecuencias que también afectarían al proceso de integración. Entre esas consecuencias lingüísticas, se incluyen los fenómenos como las interferencias, el acento o el uso alterno de lenguas (Moreno, 1990).

Las interferencias fonéticas-fonológicas pueden causar ruidos comunicacionales entre culturas, ya que pueden actuar como elemento bloqueador en el proceso de adquisición de la L2 (Durão, 2004, p. 47). La utilidad de este estudio radicaría en el hecho de que el fenómeno migratorio, en el que dos culturas se encuentran, influyéndose mutuamente, es universal; por lo que la comprensión del principal vehículo integrador como lo es la lengua debe ser objeto de estudio minucioso. Por tanto, partimos de la importancia según la cual los resultados obtenidos en este estudio podrían ser aplicables a otras situaciones de migración que conlleven el aprendizaje de otra lengua en el país de destino.

Nuestro objetivo de investigación es clasificar las interferencias lingüísticas en el discurso oral de los inmigrantes portugueses que llegaron a Venezuela en el periodo 1950 a 1980 , conforme a la clasificación de las interferencias fonéticas-fonológicas de Porto Cardoso (2009), Niño Rojas (2007) y Obediente (2007). Para ello, trabajamos con una muestra de 30 inmigrantes de ambos sexos que llegaron a Venezuela entre los años 1950 y 1980. Realizamos una entrevista que estuvo estructurada en dos partes: la primera hace referencia a los datos personales y sociales del informante y la otra se centra en sus habilidades lingüísticas. Utilizamos como técnica para evaluar las habilidades lingüísticas los relatos de vida (Martín, 1995). 


\section{Materiales y método}

Con el propósito de recoger la información para este estudio, se utilizó la técnica de la entrevista, que incluye una parte para obtener datos sociodemográficos de elaboración propia, usando como modelo las líneas metodológicas de PRESEEA (2003) y la propuesta de Moreno (2009). El cuestionario fue administrado por un entrevistador en visita privada. Los datos fueron organizados en una tabla de datos sociodemográficos.

Para recoger información sobre los relatos de vida, se realizó una entrevista individual semi-abierta de elaboración propia, estructurada en cinco preguntas. Permitimos que los sujetos hablaran libremente con el fin de detectar y clasificar las posibles interferencias fonéticas-fonológicas. Las entrevistas fueron realizadas y grabadas en Venezuela y en Portugal. Como señala Moreno (1990, p. 58), la grabación de la entrevista permite catalogar fielmente el discurso oral de los migrantes. En efecto, estos relatos de vida nos permitieron observar las habilidades linguiísticas y evidenciar las posibles interferencias fonéticas-fonológicas. En relación a la duración de las entrevistas, estas variaron de 10 a 30 minutos, sin limitación de tiempo predeterminado. Para identificar las interferencias fonéticas en los relatos, seleccionamos al azar un segmento de siete minutos de cada entrevista. Observamos que la disposición emocional de los entrevistados en los relatos de vida jugó un papel importante con respecto a la confiablidad de los datos suministrados, ya que el estilo más próximo al conversacional surgía cuando el hablante se manifestaba a propósito de experiencias más emotivas (Larrosa, 2003, p. 160). Los relatos de vida tienen una función exploratoria y una función analítica (Bertaux, 1989). En su función exploratoria, los relatos de vida nos proporcionaron información biográfica de los entrevistados, y en su función analítica, conseguimos contextualizar los procesos lingüísticos (Ferrarotti, 1990, p. 41).

Posteriormente, con base a los audios grabados de los relatos de vida, se realizó una transcripción fonética-fonológica del corpus seleccionado, respetando fielmente los alófonos emitidos por los inmigrantes con el fin de poder obtener una clasificación de las interferencias fonéticas-fonológicas. Una vez obtenidas las transcripciones, se procedió a utilizar el Software Atlas ti versión 8.0, que es una herramienta informática cuyo fin es facilitar el análisis cualitativo de datos textuales y poder acceder con facilidad a los textos e identificar la frecuencia de las interferencias fonéticas-fonológicas en los relatos de vida. Pudimos constatar, con el apoyo del software Atlas ti versión 8.0, que en todos los relatos de vida (documentos primarios) ocurrió una saturación en relación a las interferencias lingüísticas. Esta saturación nos permitió identificar el momento más oportuno para detener la recolección de los datos (Strauss y Corbin, 2002). En el instante en que las comparaciones constantes entre los datos no evidenciaron nuevas relaciones o propiedades de los datos, la riqueza que representaban los datos se comenzó a agotar. Para realizar este proceso de codificación abierta, se hizo un análisis de las posibles interferencias lingüísticas a nivel fonético-fonológico.

Para describir la población estudiada, tomamos en consideración las siguientes características personales: sexo, edad, grado de instrucción y ocupación. La muestra estuvo constituida por inmigrantes portugueses, de ambos sexos, que llegaron a Venezuela entre los años 1950 a 1980 con edades comprendidas entre 10 y 30 años. Para el momento de su llegada, la mayoría contaba con un nivel escolar - primario- hasta la cuarta clase. Al recolectar los datos, los sujetos tenían entre 48 y 63 años de permanencia en el país. La mayoría de los entrevistados 
eran comerciantes los hombres y amas de casa las mujeres, provenientes en su mayoría de Portugal continental (Barcelo, Aveiro, Porto, Braga, etc.), solo algunos de la Isla de Madeira.

La muestra estuvo inicialmente constituida por 30 sujetos elegidos al azar con más de 40 años viviendo en Venezuela. Cuatro sujetos fueron excluidos de la muestra por no cumplir con los criterios de inclusión, quedando la muestra final formada por 26 individuos. Los sujetos excluidos no cumplieron con los criterios de inclusión por falta de cooperación y temores personales sobre la posibilidad de compartir sus relatos de vida. En relación al tamaño de la muestra, Castellano (2001, p. 45) plantea que la elección de los sujetos es una de las primeras dificultades con las que tropieza el investigador en sociolingüística. No existe un método uniforme para la selección cuantitativa y cualitativa de la muestra. En este sentido, SilvaCorvalán (1989, p. 20) señala que la dificultad para determinar el número óptimo de individuos en investigaciones sociolingüísticas dependerá de las características de la investigación que se vaya a llevar a cabo.

Tabla 1. Edad y sexo

\begin{tabular}{|l|c|c|c|}
\hline $\begin{array}{l}\text { Sexo } \\
\text { Edad }\end{array}$ & Masculino & Femenino & Total \\
\hline 60 a 69 años & 3 & 3 & 6 \\
\hline 70 a 79 años & 6 & 12 & 18 \\
\hline 80 a 89 años & 1 & 1 & 2 \\
\hline Total & 10 & 16 & 26 \\
\hline
\end{tabular}

La muestra quedó constituida como aparece en la Tabla 1. En relación con la variable sexo, podemos distinguir el "sexo" del "género". El primero hace referencia al sexo biológico, el segundo al sexo socio-cultural, es decir, el conjunto de elementos que constituyen un modelo social de la distinción entre sexos. Para efectos de esta investigación haremos referencia al sexo biológico. El sexo femenino representó $62 \%$ de la muestra. Esta variable resulta interesante, ya que hombres y mujeres hablan de forma diferente (Serrano, 2008, p. 176).

Con respecto a la edad actual de los sujetos, observamos que la mayoría tiene entre 70 y 79 años. Las medidas de tendencia central son coincidentes. El promedio es de 72 años, la moda es 70 años y la mediana 72 años. La coincidencia de estos valores centrales da cuenta de una buena muestra. Una observación interesante es que hay la misma cantidad de hombres y mujeres en los grupos de edad de los mayores y de los menores, excepto con el grupo de los sujetos entre 70 y 79 años en el cual la cantidad de mujeres duplica el número de hombres.

Tabla 2. Grado de instrucción y ocupación

\begin{tabular}{|l|c|c|c|}
\hline $\begin{array}{l}\text { Nivel de estudio } \\
\text { Ocupación }\end{array}$ & Sin estudio & Primaria & Total \\
\hline Ama de casa & 2 & 8 & 10 \\
\hline Comerciante & & 10 & 10 \\
\hline Herrero & & 3 & 3 \\
\hline Construcción & 1 & 2 & 3 \\
\hline Total & 3 & 23 & 26 \\
\hline
\end{tabular}


Bondarenko (2015, p. 183) explica que la principal motivación de los portugueses para emigrar era la necesidad económica, debido a una fuerte crisis y depresión en Portugal. Para los años 1950 a 1980 los portugueses emigraron a Venezuela en búsqueda de una mejor calidad de vida. De acuerdo con la Tabla 2, se observa que en su gran mayoría los sujetos contaban con al menos formación primaria en Portugal. Ya en Venezuela no completaron sus estudios, puesto que no tenían los medios para continuar una formación académica. Ninguno de los informantes manifestó tener alguna profesión en el momento de su llegada a Venezuela. El nivel de aprendizaje del español pudo estar influenciado por el nivel de estudio de los inmigrantes, los cuales aprendieron el español a través del contacto diario y espontáneo con la gente en los lugares de trabajo y de residencia. Parte de ese proceso consistía en recibir explicaciones informales de amigos hispanohablantes en cuanto a la pronunciación y a la escritura, además de leer y comprender la prensa venezolana (Tavares, 2006). El nivel de estudio y las similitudes lingüísticas de ambas lenguas jugaron un papel importante a la hora de la adquisición del español. Ambas lenguas (portugués y español) presentan características similares, de manera que el proceso de aprendizaje de la nueva lengua pudo ser mucho más fácil y llevadero para el inmigrante, pues se le presenta un universo que ya conoce o que al menos le es familiar (López, Quesada y Salas, 2014, p. 5).

Es interesante resaltar que para el año 1956, en Portugal la escolaridad era obligatoria hasta los estudios primarios: los del sexo masculino llegaban hasta la $4^{\text {a }}$ clase y el sexo femenino hasta la $3^{\text {a }}$ clase (Fraser, 1993, p. 12). Para los años cincuenta, Portugal presentaba una alta tasa de analfabetismo producto de la fuerte crisis económica, política y social, derivada del impacto de la participación de Portugal en la I Guerra Mundial, por lo que, según algunos autores, el gobierno comenzó a tomar medidas relacionadas con la escolaridad para resolver este problema crónico (Ferreira, 2011, p. 13).

En la Tabla 2 podemos observar que $38 \%$ de los entrevistados se dedica al comercio. Un $12 \%$ de la muestra trabaja en la construcción, un $12 \%$ de los entrevistados trabaja en la herrería y un $38 \%$ de la muestra son amas de casa. Tal como plantea Moreira (1998), los portugueses se instalaron en Venezuela y se dedicaron al comercio, la agricultura, la pesca, la construcción y a los oficios manuales como la zapatería o la carpintería. En la muestra en cuestión, los hombres son los encargados de proveer el sustento económico, mientras que las mujeres son las responsables de la familia y de los quehaceres domésticos. Es interesante resaltar que los portugueses en Venezuela tienen unos ingresos de más de 500 euros mensuales, muy superior al promedio actual del venezolano (50 euros mensuales), y gracias a ello, han tenido un papel muy importante en la economía del país. Gomes (2009, pp. 86-87) refiere que:

No país, os portugueses são os proprietários de aproximadamente $80 \%$ do total das padarias e $60 \%$ do total das mercearias. No sector agrícola os portugueses têm tido um papel muito importante ao nível da produção e distribuição. Neste sector destacam-se as produções de cana-de-açúcar, arroz, mandioca, batata, tomate e frutos, em especial a banana e a laranja. No sector da pecuária, embora em menor escala, a criação de gado bovino e a criação de suínos cochineras encontram-se nas mãos de alguns portugueses, especialmente nos estados de Miranda, Arágua e Lara².

2 En el país, los portugueses son propietarios de aproximadamente $80 \%$ del total de las panaderías y 60 $\%$ del total mercerías. En el sector agrícola los portugueses han tenido un rol muy importante a nivel de la producción y distribución. En este sector se destacan las producciones de caña de azúcar, arroz, yuca, papa, tomate y frutas, en especial la banana y la naranja. En el sector pecuario, aunque en menor escala, la cría de ganado bovino y porcino "cochineras" están en manos de algunos portugueses, especialmente en los estados de Miranda, Aragua y Lara (Gomes, 2009, pp. 86-87, traducción propia). 
Una vez más, estos datos arrojados en la investigación confirman lo planteado por diversos autores (Moreira, 1998; Tavares, 2006 y Bondarenko, 2015): que los portugueses se instalaron en tierras venezolanas y con frecuencia fueron ellos los que dieron empleo a los venezolanos, con lo que se logró un proceso de integración social entre ambas culturas. Podemos suponer que el grado de interferencias lingüísticas de los inmigrantes no entorpece en este caso la integración social de los portugueses en Venezuela.

En cuanto a la percepción de los hablantes, en las conversaciones con los inmigrantes, la mayoría no discierne en el habla cuando ocurren las interferencias. A continuación, señalamos en los resultados las interferencias más recurrentes encontradas en los relatos de vida de los inmigrantes portugueses en Venezuela.

\section{Resultados}

En relación al análisis de las grabaciones de relatos de vida de los inmigrantes portugueses llegados a Venezuela durante los años 1950 a 1980, podemos identificar diferentes interferencias fonéticas-fonológicas. Los resultados se presentan en dos partes: una primera, donde se observan tres figuras que muestran las redes semánticas más frecuentes según el tipo de interferencia, ya sea por incrementación, por reducción o por sustitución; y en una segunda parte, se resume en tres gráficos de porcentajes la frecuencia de las interferencias observadas en cada uno de los tipos.

\subsection{Redes semánticas según interferencias fonéticas-fonológicas}

Las interferencias fonéticas-fonológicas las clasificamos según lo propuesto por Cañada (1994), Obediente (2007), Niño (2007) y Hualde (2014). Las interferencias ocurrieron por: incrementación (prótesis, epéntesis y paragoge), reducción (aféresis, síncopa, apócope y elisión) y sustitución de fonemas.

Específicamente, presentamos tres redes semánticas en relación con los diferentes fenómenos (incrementación, reducción y sustitución) encontrados en los relatos de vida.

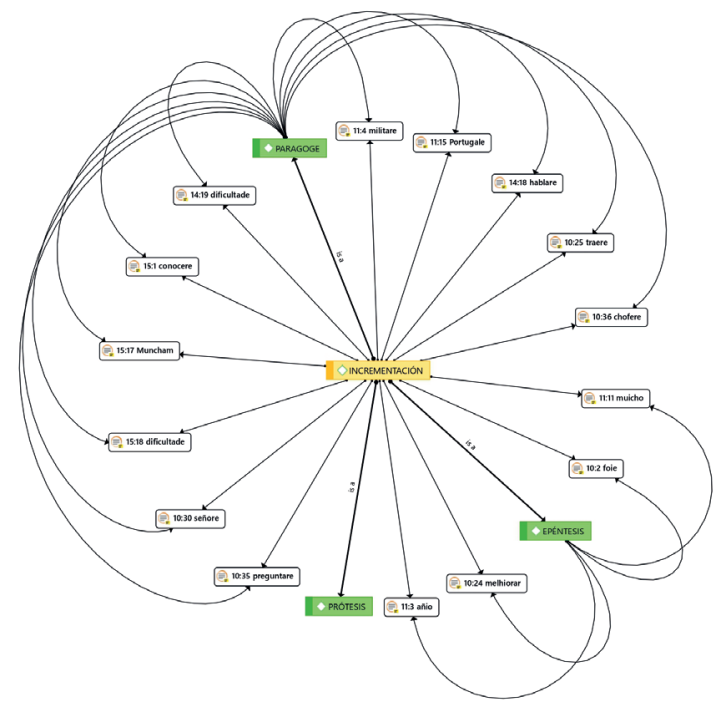

Figura 1. Incrementación fonemas 
En la categoría de Incrementación, observamos en la Figura 1 que las interferencias se dieron por epéntesis y por paragoge, en cambio no se observó ninguna por prótesis.

\section{Prótesis}

La prótesis es definida por Obediente (2007, p. 194) como el desarrollo, al inicio de una palabra, de un elemento no etimológico con el fin de hacer más fácil la pronunciación. Ninguno de los sujetos presentó fenómeno de prótesis, es decir, el incremento de un fonema o vocablo a principio de palabra.

\section{Epéntesis}

Para Obediente (2007, p. 195), este fenómeno consiste en intercalar en una palabra o un grupo de palabras un sonido no etimológico por razones de eufonía, de comodidad articulatoria, por analogía. El fenómeno de incrementación epéntesis es la inserción de un segmento. El elemento epentético puede ser una vocal o una consonante (Hualde, 2014, p. 105). Los evidenciamos en los relatos de vida: añio, melhiorar, muicho. Este hecho puede interpretarse porque los inmigrantes portugueses en Venezuela al hablar el español, lengua con similitudes lingüísticas al portugués, tienden a transferir elementos de la L1 a la L2. Por ejemplo, palabra del español mucho con la palabra del portugués muito. La teoría del desarrollo cognitivo de Piaget dice "constructivista" donde construimos nuevos conocimientos a partir de conocimientos ya existentes en nuestro cerebro (Toro, s. f.).

\section{Paragoge}

Las interferencias en los relatos de vida ocurren con la inserción de un segmento al final de la palabra, este fenómeno es la paragoge (Hualde, 2014, p. 318). Tales casos lo encontramos en palabras como: militare, chofere, conocere, hablare. Podemos suponer que esta interferencia fonética de incrementación de fonema vocálico /e/ a final de palabra puede deberse al hecho de que en portugués ciertas palabras como dificultad se escribe y pronuncia dificultade. Asimismo, en el portugués de Portugal es común el incremento de /e/ al final de los infinitivos (Porto, 2009, p. 75). Los inmigrantes portugueses entrevistados mantienen de forma inconsciente estructuras del portugués en el español, ya que los esquemas lingüísticos de la L1 sirvieron de guía en un primer momento para la producción de la L2, sin embargo, esta incorporación persiste y fosiliza la competencia de la L2, por lo que podemos hablar de una fosilización (Simoes, 2016, p. 111).

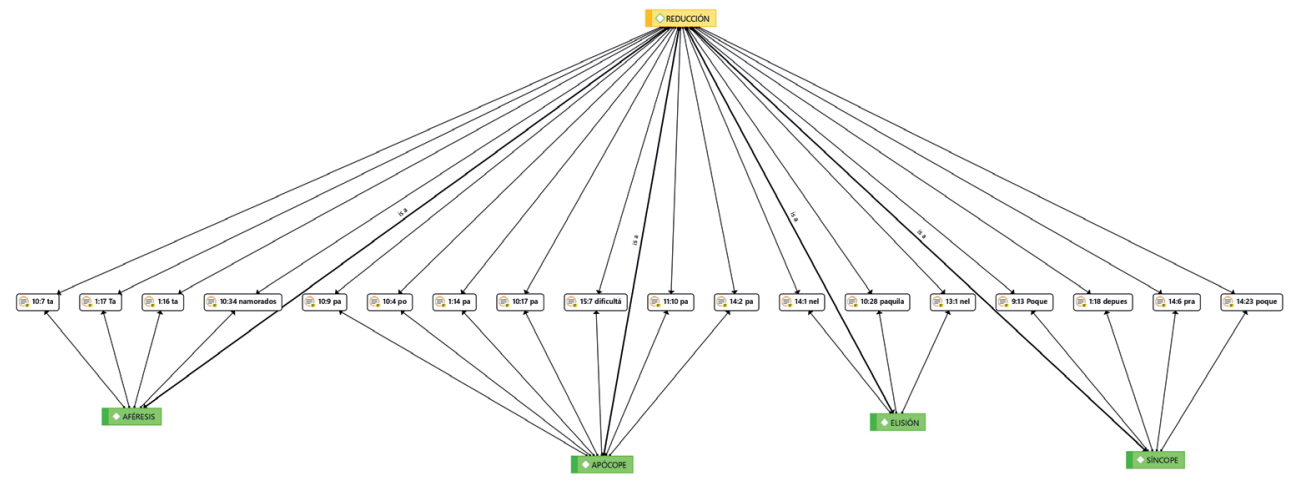

Figura 2. Reducción de fonemas

La reducción o pérdida de fonemas se dio mediante los siguientes procesos: 


\section{Apócope}

Como podemos evidenciar en la Figura 2, el fenómeno más recurrente fue la apócope, esto es que un fonema o vocablo se elimina a final de palabra. Para Obediente (2007, p. 194), este fenómeno sucede si la pérdida de elementos ocurre a final de palabra. En los relatos de vida de los inmigrantes lo evidenciamos en palabras como: $p a^{\prime}, p o^{\prime}$, dificultá. La existencia de este fenómeno no es un indicador de la presencia de interferencias fonéticas, ya que son anomalías propias del habla del venezolano. En portugués también ocurre el apócope de para, tanto en el lenguaje oral como en el lenguaje escrito. Al respecto, Rodrigues de Souza (2002, p. 39) explica que el "apócope é também processo de simplificação silábica e vocabular comum a todas as variedades do português em geral"3.

\section{Aféresis}

El fenómeno de aféresis es aquel en el que se retiran fonemas o sílabas al principio de la palabra (Porto Cardoso, 2009, p. 75). Para Obediente (2007, p. 194) esto ocurre si la pérdida tiene lugar en la parte inicial de la palabra, como en: 'ta, namorados. En este caso, el verbo estar, modo indicativo, tiempo presente, en $3^{\circ}$ persona del singular, tanto en el español como en el portugués, se conjuga como está. En ambas lenguas ocurre el fenómeno aféresis. Rodrigues de Souza (2002, p. 38) menciona que la "aférese é processo de alta frequiência nas variedades do português em geral"4. En el caso de la palabra enamorados en portugués se dice namorados.

\section{Elisión}

En el español, la agrupación de vocales heterosilábicas en la cadena sonora del discurso se resuelve generalmente por sinalefa. Obediente (2007, p. 195) entiende por sinalefa la reducción silábica por formación, entre palabras, de un diptongo (o triptongo), allí donde, según las normas, debiera haber hiato. En relación al fenómeno de elisión, se presentaron interferencias entre ambas lenguas. Observamos que los inmigrantes expresaron palabras como: nel, paquila. En el caso de la palabra nel ocurre una supresión de uno o más fonemas de una frase que sería en el. En el caso de la estructura lingüística del portugués, conseguimos la contracción de la preposición em con el artículo ele que se transforma en no. Simoes (2016, p. 179) explica que las contracciones con los artículos son otro problema de los lusohablantes, ya que hay muchas en portugués. En español solo existen dos contracciones: las que se producen con las preposiciones "de" y "a" y el artículo definido "el" (del y al). Será frecuente en los niveles elementales el hecho de que los lusohablantes utilicen contracciones y combinaciones que, por lo general, provienen del portugués, o bien puede suceder que intenten traducirlas al pie de la letra del portugués al español. Tal es el caso de la palabra nel o en el caso de la palabra paquila, se elide la preposición para con el pronombre demostrativo aquella. En portugués, el pronombre demostrativo femenino singular aquella se escribe aquela y en algunos regiones de portugal se pronuncia [akila].

\section{Síncopa}

Para Obediente (2007, p. 195), la síncopa es la pérdida de un sonido que se produce dentro de la palabra. Los casos más relevantes en los relatos de los inmigrantes son: poque,

3 “apócope es también un proceso de simplificación silábica y de vocabulario común a todas las variedades de portugués en general” (Rodrigues de Souza, 2002, p. 39, traducción propia).

4 "La aféresis es un proceso de alta frecuencia en las variedades del portugués en general" (Rodrigues de Souza, 2002, p. 38, traducción propia). 
depues, pra. Podemos decir que este fenómeno ocurre tanto en hisponohablantes como en lusohablantes al usar un lenguaje informal. Rodrigues de Souza (2002, p. 38) explica que este es un proceso común, resultado de un mecanismo de simplificación de las lenguas. Por tanto, las causas de este cambio pueden deberse a errores espontáneos de pronunciación.

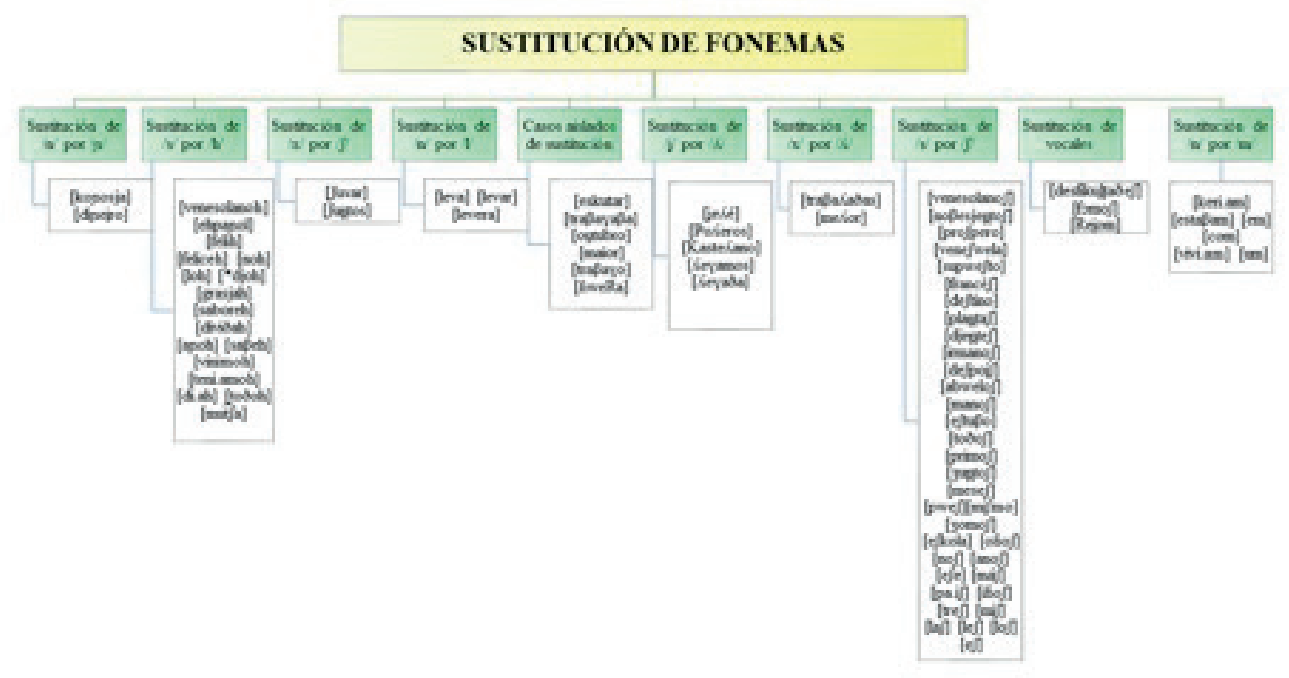

Figura 3. Sustitución de fonemas

Podemos observar en la Figura 3 que los fenómenos de sustitución fueron los más frecuentes en los relatos de vida de los inmigrantes, según la clasificación propuesta por Cañada (1994). La mayoría de los fenómenos fonéticos de sustitución dan origen a interferencias fonéticas-fonológicos entre el español y el portugués como presentaremos a continuación.

La sustitución más recurrente es la del fonema fricativo alveolar sordo /s/ en posición postnuclear por el fonema fricativo postalveolar sordo $/ \int /$ en posición postnuclear. Las palabras más frecuentes fueron venezolanosh, puesh, primosh, manosh, deshtino, proshpero, todosh, Venezhuela, dientesh. Podemos decir que esta sustitución es una interferencia fonética porque en el repertorio fonológico portugués existe el fonema / / y por el contrario en el español no existe este sonido. Tavares (2006, p. 112) explica que el fonema /s/ postnuclear -o codapronunciado con la fricativa postalveolar sorda [ $\left.\int\right]$ es el rasgo más productivo y, por ende, el más reconocible por los hablantes hispanos. En muchos casos llega a fosilizarse en numerosos hablantes, especialmente en aquellos que han inmigrado con mayoría de edad.

Es frecuente escuchar en el habla de los venezolanos el fonema fricativo alveolar sordo /s/ en posición postnuclear como el fonema fricativo glotal sordo /h/, es decir, se glotaliza el fonema /s/ en posición postnuclear. Este fenómeno Quilis y Fernández (2003, p. 97) lo denominan aspiración, ocurre cuando el fonema /s/ en posición posnuclear, no se realiza como [s], sino que se aspira, realizándose como una fricativa laríngea [h], fenómeno ocurrido en amplias zonas de España y de Hispanoamérica. Específicamente, en el español de Venezuela, Obediente (2007, p. 179) expone que el fonema fricativo alveolar /s/ se realiza en tensión silábica comúnmente como predorso-alveolar [s] y en distensión silábica como [s], aspirada $[\mathrm{h}]$ o puede elidirse totalmente. En el caso los diferentes relatos de los portugueses, 
pudimos observar esta aspiración en las palabras: díaj [dí.ah], muchoj [mutfoh], teníamoj [tení.amoh], saborej [saßoreh], venezolanoj [benesolanoh], graciaj [grasjah], Dioj [*djoh], añoj [anoh]. Podemos decir que esta sustitución no es una interferencia fonética, sino una adopción del fenómeno ocurrido por el uso frecuente del idioma. Este fenómeno de aspiración ha sido reportado tanto en España como en América (Villamizar, 1998, p. 47).

En los relatos encontramos inmigrantes que tienden a bilabializar [m] en el fonema nasal alveolar $/ \mathrm{n} /$ en posición postnuclear, como en las siguientes palabras: em, com, um, vivíam, estabam, queríam. Esta pronunciación bilabializada la podemos categorizar como una interferencia fonética, ya que el único caso en el que el fonema nasal alveolar sonoro se bilabializa en español es cuando tienen por delante los fonemas /p/ y /b/. Este hecho puede deberse a que, en portugués, las preposiciones: en, con; el artículo indeterminado un; nombres comunes como hombre y verbos en $3^{\circ}$ persona del plural terminan con el fonema nasal bilabial sonoro $/ \mathrm{m} /$ y no en el fonema nasal alveolar sonoro $/ \mathrm{n} /$. Por consiguiente, se produce una fosilización, ya que el sistema fonológico de la mayoría de los portugueses llegados a Venezuela está consolidado y difícilmente lograron establecer nuevas categorías fonéticas y fonológicas, provocando que su adquisición fónica no avance (Férriz, 2001).

Según Peña (2015, p. 192), la totalidad del territorio venezolano es plenamente yeísta. Entiéndase por yeísmo la sustitución del fonema fricativo palatal sonoro /j/ por el fonema líquido lateral palatal sonoro $/ K /$. Según la RAE (2017), yeísmo es la desaparición de la diferencia fonológica entre la consonante lateral palatal y la fricativa palatal sonora, de manera que, en la pronunciación, no se distinguen palabras como callado y cayado. Este cambio fonético propio del venezolano no lo observamos en la pronunciación de los inmigrantes, esto puede deberse a que el portugués tiene el sonido $/ K /$ con la grafía $l h$. Las palabras más frecuentes en los inmigrantes fueron: castelhano [kasteKanu], lhegamoj [Keyamof], lhegadah [Кеуаða]], polheros [poКerof].

En cuanto a los sonidos vocálicos, el español cuenta con cinco vocales puras. El portugués tiene esas cinco y siete adicionales. Por lo tanto, los inmigrantes al momento de hablar tienden a usar los fonemas vocálicos del portugués y no hacen una discriminación con los fonemas vocálicos del español. Tal es el caso del sonido $e$ que suena como /e/ y el portugués tiene esta y una más, como la (e) abierta /ع/. Es difícil predecir cuál usar en cada palabra. Asimismo, Tavares (2006, p. 112) menciona que la elevación de la vocal /o/ a [u] es un fenómeno particular que en portugués también es importante y se produce cuando el fonema es átono. Simoes (2016) explica que el lusohablante distingue entre vocales abiertas y cerradas cuando habla español. Las vocales $e$ y $o$ tienden a nasalizar y a cerrar la "e" y la "o" en determinadas posiciones dentro de la palabra, por ello les costará pronunciar estas vocales sin cerrarlas hasta convertirlas en el sonido [i] y [u]. En los relatos encontramos palabras como castellano, por ejemplo, la cual pronuncian como si fuera una [u]: [kasteKanu]. Los sonidos vocálicos en español tienen pronunciación igual entre los hispanohablantes y su variedad de sonidos es menor que en portugués.

\subsection{Frecuencia de las interferencias}

La frecuencia de las interferencias podemos resumirlas de la siguiente manera: 


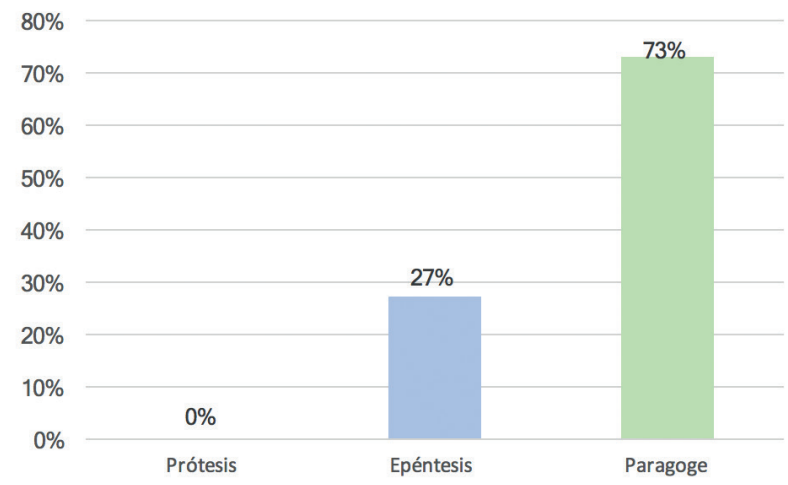

Figura 4. Interferencia por incrementación de fonemas

En las interferencias fonéticas-fonológicas por incrementación, podemos observar en la gráfica de la Figura 4 que el error más frecuente, con un $73 \%$, fue la paragoge, es decir, que ocurre un incremento de uno o varios fonemas a final de palabra.

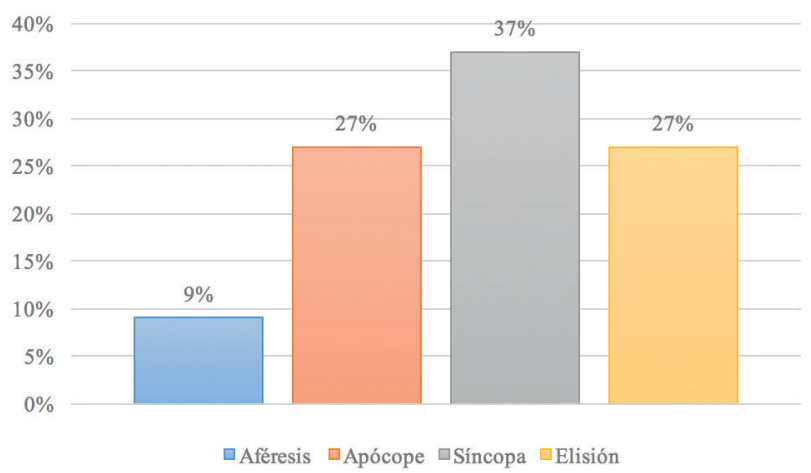

Figura 5. Interferencias por reducción de fonemas

Como podemos observar en la gráfica de la Figura 5, la interferencia por reducción más frecuente producida por los portugueses en los relatos de vida fue el de síncopa con $37 \%$, es decir, de la omisión o supresión de uno o varios fonemas a mitad de palabra.

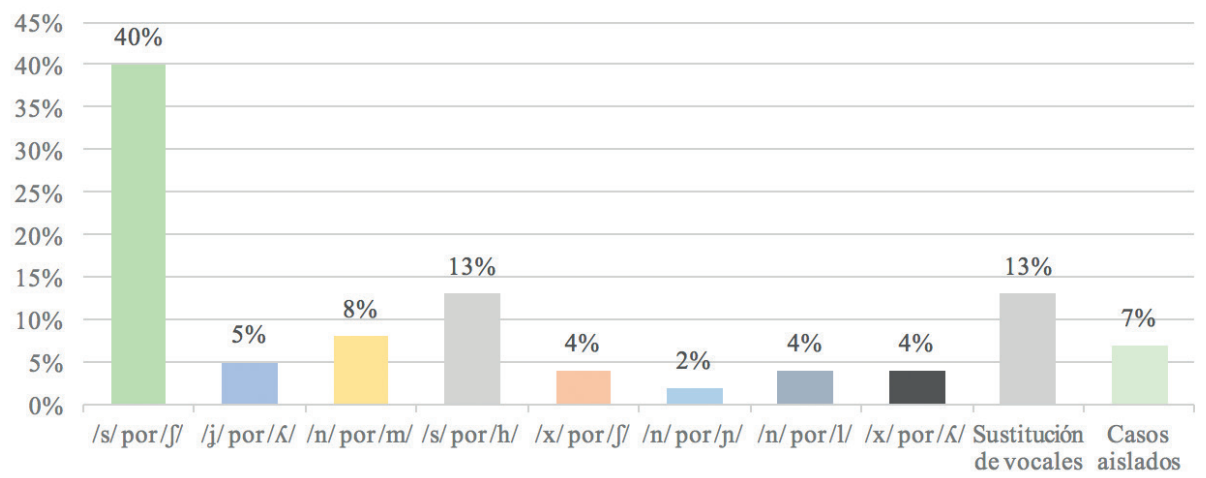

Figura 6. Interferencia por sustitución de fonemas 
Como se evidencia en la Figura 6, en el caso de las interferencias ocurridas por la sustitución de fonemas, la más significativa fue la sustitución del fonema fricativo alveolar sordo /s/ por el fonema fricativo postalveolar sordo / $/$ / con una frecuencia de $40 \%$. Con un 13 $\%$ observamos tanto la sustitución de vocales como el fenómeno de glotalización del fonema fricativo alveolar sordo /s/ en posición postnuclear $/ \mathrm{h} /$.

El rasgo más significativo fue el de la pronunciación del fonema fricativo postalveolar sordo / / en posición posnuclear, fenómeno corriente en el portugués europeo. Tavares (2006, p. 112), en su investigación acerca del contacto lingüístico entre el español y el portugués: caso de inmigrantes portugueses radicados en Venezuela, mencionó que el fonema / $/$ es el rasgo más productivo y en muchos casos llega a fosilizarse en numerosos hablantes, especialmente en aquellos que han inmigrado con mayoría de edad. Esto puede estar relacionado con el hecho de que la comunidad portuguesa interactúa con el venezolano sobre todo en el aspecto laboral (comercial). En cuanto a la vida social de estos inmigrantes, generalmente la hacen dentro de su mismo grupo cultural. Asimismo, según Tavares (2006, p. 109) este grupo humano forma parte de la clase media, cuyas ocupaciones están vinculadas, en su mayoría, con el sector comercial.

\section{Conclusiones}

Las interferencias más significativas en los relatos de vida de los inmigrantes fueron las siguientes: en los errores por reducción, el más frecuente fue la síncopa con un $37 \%$ de frecuencia. En el error por incrementación, el más frecuente fue la paragoge con un $73 \%$. Finalmente, el error por sustitución más utilizado en los relatos por los portugueses, con un $40 \%$ de frecuencia, fue el de la sustitución del fonema fricativo alveolar sordo /s/ por el fonema fricativo postalveolar sordo / $/$ / Por tanto, a partir del estudio de este corpus de relatos de vida, podemos constatar que los portugueses producen una serie de anomalías al momento de expresarse en la L2, ya que hay un bloqueo en la producción fluida del español, quizás por las similitudes lingüísticas entre ambas lenguas, causando que las transferencias de la L1 fosilizasen la L2. En este sentido, Vázquez (1992, p. 107) argumenta que la transferencia suele ser la principal causa de los errores fosilizables y fosilizados.

Diversos autores (Andrade, 2000; Almeida, 2001; Álvarez, 2002; Celada, 2002; Camorlinga, 2005; Salinas, 2005; Simoes, 2016; Lafuente, 2017) concuerdan con que uno de los motivos para el estancamiento podría ser la proximidad entre el español y el portugués y que esta proporciona beneficios para el aprendizaje en un principio, pero se torna una dificultad en los estadios más avanzados.

Es importante mencionar que el $88 \%$ de los portugueses estudiados hablan "portuñol". Entiéndase este término como la mezcla del español y del portugués de forma compartida. Esta anomalía tiene su origen en el parecido de ambas lenguas. Ferreira (1995, p. 39) define el "portunhol" como "la expresión más inmediata del contacto entre las lenguas portuguesa y española y que puede ser descrita como una interlengua con niveles de fosilización muy avanzados".

Las interferencias fonéticas-fonológicas más recurrentes fueron las de sustitución de vocablos con un $55 \%$ de frecuencia, las de reducción de fonemas con $25 \%$ y finalmente, con un $20 \%$ la incrementación de sonidos. Todo esto nos lleva a pensar que, en un primer contacto con la lengua española, los portugueses inmigrantes mejoraron su integración en la cultura venezolana debido a las semejanzas lingüísticas que presentan las dos lenguas. 
Pero, debido a la falta de formación académica, estos no pudieron aprender formalmente el español, acarreando con esto que los inmigrantes fosilizaron la L2, es decir, quedaron elementos sonoros del portugués en el español. Según diversos autores, Almeida (2001, p. 18), Kulikowski y González (1999, p. 13), parece haber consenso en que los errores causados por interferencia tienden a fosilizarse. De este modo, se produjo un estancamiento o bloqueo en la evolución de la lengua y en la producción fluida del español entre los inmigrantes portugueses que fueron a Venezuela en el periodo estudiado.

Se evidencia una fosilización en la L2, lo que pudiera ser explicado por diversos factores relevantes. Un factor pudo haber sido la edad al emigrar, en su mayoría, los sujetos se encontraban en la adolescencia. Otro factor podría haber sido el grado de instrucción de los inmigrantes, quienes no fueron escolarizados en el sistema educativo venezolano. Si bien las similitudes lingüísticas entre el portugués y el español facilitaron, en un primer momento, la comprensión del español, los inmigrantes no lograron formalizar su aprendizaje. Es importante recalcar que los inmigrantes pueden hacerse entender perfectamente a pesar de las interferencias fonéticas por incrementación, reducción y sustitución.

\section{Bibliografía}

Abreu, M. (2011). Análise sociolinguística de interferências fonético-fonológicas no português brasileiro de contato. Signum: Estudos da Linguagem, 14(2), 375-395.

Almeida Filho, J. (2001). Uma metodologia específica para o ensino de línguas próximas? En J. C. P. Almeida Filho (Ed.), Português para estrangeiros interface com o espanhol (pp. 13-21). Campinas: Pontes.

Álvarez, M. (2002). La transferência, la interferência y la interlengua en la enseñanza de lenguas próximas. Scielo. Brasilia: Universidade de Brasilia. Recuperado de http:// www.proceedings.scielo.br/scielo.php?pid=MSC0000000012002000100039\&script= sci_arttext

Andrade Neta, N. (2000). Aprender español es fácil porque hablo portugués. Ventajas y desventajas de los brasileños para aprender español. Cuadernos Cervantes de la lengua española, 6(29), 46-56.

Bertaux, D. (1989). Los relatos de vida en el análisis social. Historia y Fuente Oral. Recuperado de http://www.jstor.org/stable/27753230

Bondarenko, N. (2015). Estudio comparativo de los aportes de las inmigraciones italiana y portuguesa. Humania del Sur, 10(18), 173-190.

Blanco, R. (2002). Una propuesta fonética didáctica para brasileños estudiantes de español. La pronunciación es la tarjeta de presentación de la expresión oral. Frecuencia L, 21, 24-28.

Brisolara, L. (2011). La interferência do sistema consonantal português no uso do español. Signum: Estud. Ling., Londrina, 14(2), 165 -182.

Cañada, M. (1994). Análisis acústico de realizaciones laterales en español y en francés: una contribución al estudio del bilingüismo en el plano fónico. (Tesis Doctoral). Universidad Autónoma de Barcelona, Barcelona. 
Cambrussi, M., Ferreira, E. y Gritti, L. (2009). Análise multilinear da interferência fônica no português de contato com o italiano. PAPIA-Revista Brasileira de Estudos do Contato Linguístico, 18(1), 71-81.

Camorlinga, R. (2005). Do português ao espanhol: os prós e os contras da proximidade. En Sedycias, J. (Ed.), O ensino do espanhol no Brasil. Passado, presente, futuro (pp. 195-205). São Paulo: Parábola.

Castellano, A. (2001). Aspectos sociolingüísticos de la variación gramatical. Gran Canarias: Universidad de las Palmas de Gran Canaria, Servicios de Publicaciones.

Celada, M. (2002). O espanhol para o brasileiro: uma língua singularmente estrangeira. (Tesis doctorall). Instituto de Estudos da Linguagem. Universidade Estadual de Campinas.

Durão, A. (2004). Análisis de Errores en la interlengua de brasileños aprendices de español $y$ de españoles aprendices de portugués. Londrina: Eduel.

Elsaadani, A. (2016). Analyse des interférences phonologiques entre l'arabe et le français (étude contrastive). NORSUD Revue bilingue semestrielle du Département de Français Faculté des Lettres - Université de Misurata - Libye, 8, 1-25.

Ferrarotti, F. (1990). Histoire et histoires de vie: la méthode biographique dans les sciences sociales. Paris: Méridiens Klincksieck.

Ferreira, I. (1995). A interlíngua do falante de espanhol e o papel do professor: aceitação tácita ou ajuda para superá-la? En J. Almeida Filho (Ed.), Português para estrangeiros interface com o espanhol (pp. 39-47). Campinas: Pontes.

Ferreira, A. (2011). Novos rumos da educação no Estado Novo: influência da abertura da economia portuguesa no pós-II Guerra Mundial no sistema de ensino português. XXXI encontro da associação portuguesa de história económica e social, APHES. Coimbra.

Férriz, Ma . (2001). Fonología contrastiva del portugués y el castellano: una caracterización que aprenden portugués. (Tesis doctoral). Barcelona: Universitat Autònoma de Barcelona.

Fraser, R. (1993). La historia oral como historia desde abajo. Ayer, (12), 79-92. Recuperado de http://revistaayer.com/articulo/1138

Gomes, N. (2009). Os portugueses na Venezuela. Relações Internacionais, 24, 83-92. Recuperado de http://www.scielo.mec.pt/scielo.php?script=sci_ arttext\&pid=S1645-91992009000400010

González-Ordosgoitti, E. (1991). En Venezuela todos somos minoría. Nueva Sociedad, 111, 128-140. Recuperado de https://www.nuso.org/media/articles/downloads/1968_1.pdf

Godoy, E., Moreira, E., Rodríguez, R. y Roppel, A. (1994). Contribuciones para la enseñanza de la pronunciación española. II Seminario de Dificultades Específicas de la Enseñanza del Español a Lusohablantes (pp. 203-205). São Paulo: Consejería de Educación de la Embajada de España en Brasil.

Hualde, J. (2014). Los sonidos del español: Spanish Language edition. Cambridge: University Press. 
Kulikowski, M. y González, N. (1999). Español para brasileños. Sobre por dónde determina la justa medida de una cercanía. Anuario brasileño de estudios hispánicos, 9, 11-20.

Lafuente, S. (2017). La intererencia como principal fuente de errorres lingüísticos en docentes brasileños de ELE: caracterización y estudio de los errores léxicos. (Tesis doctoral). Florianópolis/Castellón de la Plana: Universidad Federal de Santa Catarina y Universitat Jaume I.

Larrosa, M. (2003). Metodología sociolingüística. Anuario de lingüística hispánica, 19, 141178. Recuperado de http://uvadoc.uva.es/handle/10324/9521

López, R., Quesada, Ma . y Salas, J. (2014). Factores sociales en el aprendizaje de un segundo idioma: el caso de la sede del pacífico de la Universidad de Costa Rica. Revista de Lenguas Modernas, 20, 435 -451.

Malaca, J. y Reis, S. (2007). A Intercompreensão entre o Português e o Espanhol: Diferenças fonético-fonológicas e Lexicais. Diálogos em Intercompreensão-Colóquio Internacional. Lisboa.

Martín, A. (1995). Fundamentación teórica y uso de las historias y relatos de vida como técnicas de investigación en pedagogía social. Aula, 7, 41- 60. Recuperado de https:// gredos.usal.es/jspui/bitstream/10366/69201/1/Fundamentacion_teorica_y_uso_de_ las_hist.pdf

Moreno, F. (1990). Metodología sociolingüística. Madrid: Gredos.

Moreno, F. (2009). Integración sociolingüística en contextos de inmigración: marco epistemológico para su estudio en España. Lengua y migración (pp. 121-156). Recuperado de http://lym.linguas.net/Download.axd?type=ArticleItem\&id=23

Moreira, J. (1998). Viagem à Venezuela. Caracas: Ex Libris.

Niño, V. (2007). Fundamentos de semiótica y lingüística. Bogota: Ecoe ediciones.

Obediente, E. (2007). Fonética y Fonología. Venezuela: Universidad de los Andes Consejo de publicaciones.

Peña, J. (2015). Yeísmo en el español de América. Algunos apuntes sobre su extensión. Revista de Filología, 33, 175 -199.

Porto Cardoso, D. (2009). Fonologia da língua portuguesa. São Cristóvão: Universidade Federal de Sergipe, CESAD.

PRESEEA. (2003). Metodología del "Proyecto para el estudio sociolingüístico del español de España y de América”. Recuperado de http://preseea.linguas.net/Metodolog\%C3\%ADa.aspx

Quilis, A. y Fernández, J. (2003). Curso de fonética y fonología españolas. Madrid: CSIC.

Real Academia Española (RAE). (2017). Diccionario de la lengua española. Recuperado de http://dle.rae.es/?w=diccionario

Rodrigues de Souza, U. (2002). Fonologia e Fonologia CV do Português Mato-Grossense. Polifonia, 4(04), 15-44.

Salinas, A. (2005). Ensino de espanhol para brasileiros: destacar o uso ou a forma? En J. Sedycias (Ed.), O ensino do espanhol no Brasil. Passado, presente, futuro (pp. 54-59). São Paulo: Parábola. 
Serrano, M. (2008). El rol de la variable sexo o género en sociolingüística: ¿diferencia, dominio o interacción? Boletín de Filología, 43(1), 175-192.

Silva-Corvalán, C. (1989). Sociolingüística: teoría y análisis. Madrid: Editorial Alhambra.

Simoes, C. (2016). La enseñanza del español a lusohablantes (Portugal, Brasil, Mozambique). -Estudios constractivo, análisis de errores y propuesta didáctica. (Tesis doctoral). Madrid: Universidad Complutense de Madrid.

Strauss, A. y Corbin, J. (2002). Bases de la investigación cualitativa. Técnicas y procedimientos para desarrollar la teoría fundamentada. Antioquia: Editorial Universidad de Antioquia.

Tavares, L. (2006). Contacto lingüístico entre el español y el portugués: caso de inmigrantes portugueses radicados en Venezuela. Baciyelmo (Escuela de Letras, UCAB), (1), 104-118.

Téis, D. (2007). Interferências lingüísticas bilíngües em produções escritas. Revista Trama, 3(5), $73-87$.

Toro, A. (s. f.). Analogie et interférences dans l'apprentissage des langues. Dijon: Université de Bourgogne. Recuperado de https:/www.academia.edu/24273565/Analogie_et_ interf\%C3\%A9rences_dans_lapprentissage_des_langues

Vázquez, G. (1992). El concepto de error: estado de la cuestión y posibles investigaciones. Actas del Congreso de la Lengua Española. Sevilla Instituto Cervantes.

Villamizar, T. (1998). Fonetismo. En E. Obediente (Comp.), El habla rural de la Cordillera de Mérida (pp. 27-94). Mérida: Universidad de los Andes. 